\title{
The Biochemical Study of Intermaxillary Fixation (IMF) Stress in Oral Surgery Inpatients
}

\author{
HIDENORI SAKAINO \\ Department of Oral Surgery, Kurume University School of Medicine, \\ Kurume 830-0011, Japan
}

\begin{abstract}
Summary: Although intermaxillary fixation (IMF) is performed to treat the patients with maxillary fracture, this procedure is very stressful to the patients. IMF has been reported to increase noradrenaline (NA) release in the brain and elevate plasma corticosterone contents in the rat. These changes were significantly attenuated by diazepam, an anxiolytic of the benzodiazepine family. These results suggest that IMF could greatly affect the pituitary-adrenal system as a stress. In the present study, in order to examine the influence of IMF on the human body function, we measured levels of 17-hydrocorticosteroids (17-OHCS) and 17-ketosteroid (17-KS), which are metabolites of the adreno-cortical hormone cortisol, in the urine of inpatients undergoing IMF. The subjects were requested to fill out a questionnaire on irritableness caused by IMF. In these patients, urinary 17-OHCS levels were significantly increased after IMF and well correlated to the results of the questionnaire. The finding suggested that urinary $17-\mathrm{OHCS}$ levels reflect stress related to IMF, and that such stress mainly causes an irritated feeling. Natural killer cell activity (NK activity), which is considered to be related to stress, was measured in these patients. The relationship between 17OHCS levels and NK activity was examined in reference to the results of the questionnaire. Questionnaire showed that most patients noted insomnia and an irritated feeling during IMF. To examine the influence of anxiolytic agents on stress related to IMF, an anxiolytic agent, ethyl loflazepate, was administered during IMF, and urinary $17-\mathrm{OHCS}$ levels were measured. There was no correlation between 17-OHCS levels and NK activity in the patients. Furthermore, no correlation was observed between visual analogue scale (VSA) and NK activity. Increases in 17-OHCS levels in the group treated with ethyl loflazepate, an anxiolytic of the benzodiazepine family, were significantly lower than in the untreated group. This suggests that ethyl loflazepate reduced stress responses to IMF. It has been reported that NK activity is reduced inpatients with depression or chronic fatigue syndrome. However, NK activity may not be affected by mechanical stress such as IMF. The finding that an anxiolytic agent, ethyl loflazepate, inhibited stress responses to IMF further suggests that anxiolytic drugs are very useful for treatment of irritated feeling of the patients undergoing IMF.
\end{abstract}

Key words stress, intermaxillary fixation (IMF), ethyl loflazepate, 17-hydrocorticosteroids (17OHCS), 17-ketosteroid (17-KS), natural killer cell activity (NK activity)

\section{INTRODUCTION}

Intermaxillary fixation (IMF) is a treatment modality used in oral surgery. However, it is very stressful to the patients since during the course of IMF, various restrictions in the patient's daily life, such as incon- venience in eating and speaking can occur. In order to study the stress caused by IMF, Koga et al. [1,2] performed IMF in rats, and measured levels of noradrenaline (NA) and its major metabolite, 3-methoxy4-hydroxyphenylethyleneglycol sulfate (MHPG-SO ${ }_{4}$ ) in the rat brain. They found that IMF enhanced NA 
release in the rat brain along with increased plasma corticosterone levels and adrenal gland weight, which showed that IMF greatly affected the pituitary-adrenal system as a stressor. Further, Koga et al. [3] found that increases in both MHPG-SO $\mathrm{S}_{4}$ and plasma corticosterone levels in the rats undergoing IMF were significantly attenuated by the administration of diazepam. From these results, they suggested that anxiolytic agents could be useful for relieving stress in the patients undergoing IMF.

In the present study, in order to evaluate the influence of IMF on the human body, we measured urinary levels of catecholamines (adrenaline and NA) and of 17-hydroxycorticosteroid (17-OHCS) and 17ketosteroid (17-KS), which are metabolites of the adreno-cortical hormone cortisol, in patients undergoing IMF. Simultaneously, the patients were requested to fill out a questionnaire on irritability.

Further, we measured the activity of natural killer cells (NK activity) in these patients, since natural killer (NK) cells have been reported to play a principal role in nonspecific human immunity [4,5] and it was reported that NK activity is influenced by various types of stresses [6-9]. Further, we examined the effects of ethyl loflazepate [10,11], a long-acting anxiolytic agent of the benzodiazepine family, on changes in 17-OHCS levels in patients undergoing IMF.

\section{SUBJECTS AND METHODS}

\section{Subjects}

The subjects consisted of 59 inpatients (40 males and 19 females) who underwent IMF for the treatment of fracture or deformity of the jaw at the Department of Dentistry and Oral Surgery in Kurume University Hospital. The patient age ranged from 16 to 54 years (mean, 27.2 years). NK activity was mea-

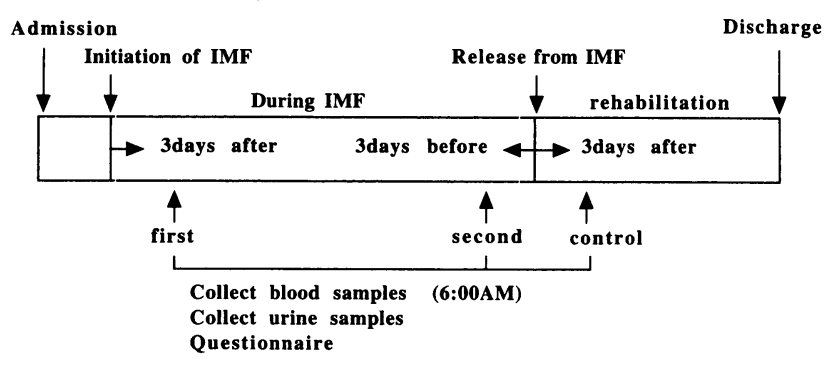

Fig. 1. Experimental schedule. sured in 19 of 59 patients, and ethyl loflazepate, a long-acting anxiolytic agent, was administered per os to 12 of 59 patients.

\section{Experimental schedule}

As in the previous study [12], a 24-hour urine sample was collected twice during the course of IMF ( 3 days after the initiation of IMF and 3 days before the release from IMF) and once 3 days after the release from IMF to measure urinary levels of 17OHCS and 17-KS. A questionnaire was filled simultaneously with the biochemical examinations twice during IMF and once after the release from IMF (Fig. 1).

NK activity was measured using blood samples collected three times early in the morning (6:00 AM) simultaneously with biochemical examinations. In addition, $1 \mathrm{mg}$ of powdered ethyl loflazepate, was administered every day once after supper during the course of IMF.

All the patients were under the stress of traumatic pain and anxiety during hospitalization. Control urine samples were collected after the release from IMF.

\section{Measurement of 17-OHCS and 17-KS}

Urine samples were collected for $24 \mathrm{hrs}$ between 0:00 a.m. and 0:00 a.m. the next day. After completion of 24-hour urine collection, $50 \mathrm{ml}$ of urine was transferred to a test tube, and stored at $-20{ }^{\circ} \mathrm{C}$ until assayed.

After dephosphorylation, a steroid conjugate of 17-OHCS was hydrolyzed using gluphatase, and then the fluorescence that developed after reaction with phenylhydrazine (Porter-Silber reaction) was measured at wave lengths of $370 \mathrm{~nm}, 410 \mathrm{~nm}$, and 450 $\mathrm{nm}$ [13]. After a similar procedure, the levels of 17$\mathrm{KS}$ were measured on the basis of the fluorescence developed after reaction with m-dinitrobenzene (Zimmermann reaction) at wave lengths of $460 \mathrm{~nm}$, $520 \mathrm{~nm}$, and $580 \mathrm{~nm}$ [14]. All data were corrected by creatinine levels.

\section{Measurement of NK activity}

Heparinized blood samples $(5 \mathrm{ml}$ each) were stored at $4{ }^{\circ} \mathrm{C}$ and $\mathrm{NK}$ activity was measured by the cytotoxicity test using the Cr-release method within 24 hrs. Briefly, the target cells (K562) were labeled with ${ }_{51} \mathrm{Cr}$, and then impaired by $\mathrm{NK}$ cells. Subsequently, the level of ${ }_{51} \mathrm{Cr}$ release from the target cells was measured $[15,16]$. We entrusted the measurement of NK activity to SRL Co., Ltd. 
TABLE 1.

Questionnaire

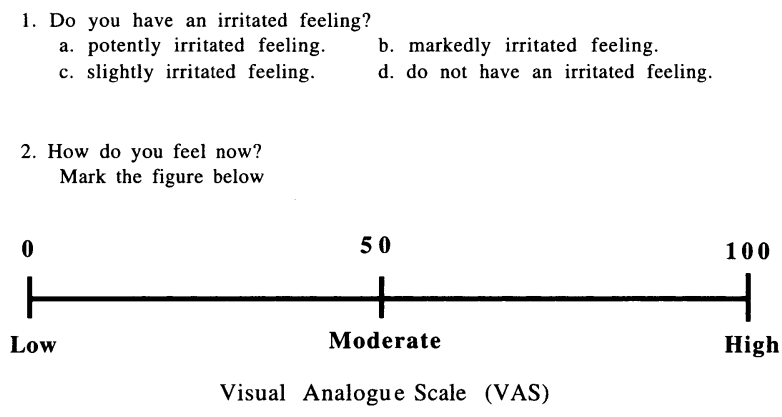

\section{Questionnaire}

Table 1 shows the details of questionnaire. In question 2, the feeling of the patients during the examinations was numerically expressed using the visual analogue scale (VSA).

\section{Statistical analysis}

Statistical analysis was performed using the analysis of variance (ANOVA). Correlation analysis was performed using Pearson's correlation analysis $[17,18]$.

\section{RESULTS}

Relationship between 17-KS and 17-OHCS levels and VAS values

The correlation between the VAS values, that reflect the feeling, and the levels of 17-KS and 17OHCS was evaluated in the 59 patients used in this study. There was positive correlation between 17OHCS $(r=-0.524)$ levels and between VAS values and 17-KS levels $(r=-0.492)$ (Figs 2 and 3). In patients not treated with ethyl loflazepate, the 17OHCS levels obtained in the first and second measurements were significantly higher than the control values ( $p<0.001$, Fig. 4 A), whereas only the levels of $17-\mathrm{KS}$ obtained in the first measurement were significantly higher than the control value $(\mathrm{p}<0.01$, Fig. 4B).

Relationship between NK activity and levels of 17OHCS and 17-KS

Mean values for NK activity in 19 patients were $28.42 \% 3$ days after the initiation of IMF, $34.11 \% 3$ days before the release from IMF, and $28.74 \% 3$ days after the release from IMF. That is, the mean

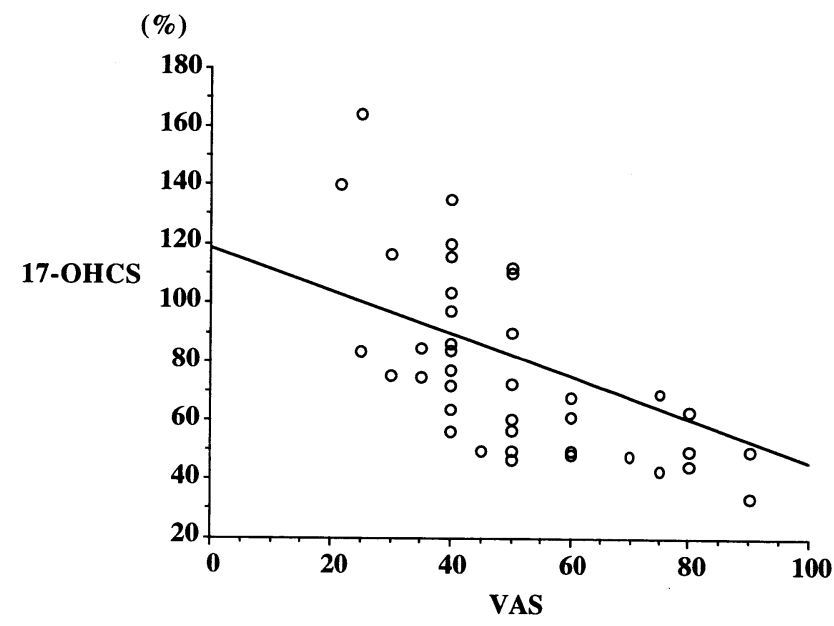

Fig. 2. Correlation between VAS values and 17OHCS levels. Correlation: $r=-0.524(p<0.001)(n=59)$

(\%)

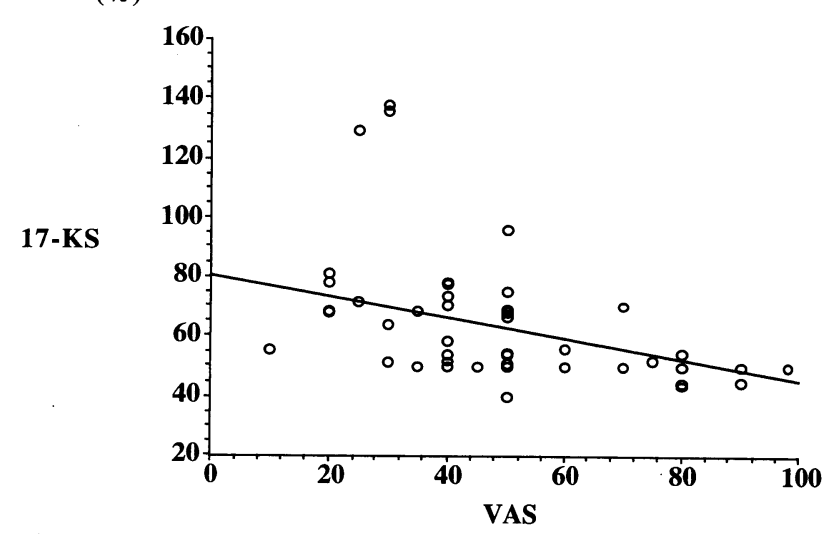

Fig. 3. Correlation between VAS values and 17-KS levels. Correlation: $\mathrm{r}=-0.492(\mathrm{p}<0.05)(\mathrm{n}=59)$

value for NK activity obtained 3 days after the initiation of IMF was similar to that obtained 3 days after the release from IMF, and both were within the range of standard values $(18-40 \%)$. There were no significant correlation between 17-OHCS levels and NK activity ( $r=0.1$ ) (Fig. 5) or between 17-KS levels and NK activity $(r=0.088)$. There was no significant correlation between VAS values and NK activity $(r=0.269)$ (Fig. 6).

\section{Effects of the ethyl loflazepate administration}

The levels of 17-OHCS were significantly lower $(p<0.01)$ in the ethyl loflazepate-treated group than in the untreated group 3 days after the initiation of IMF (Fig. 7). 
(\%)

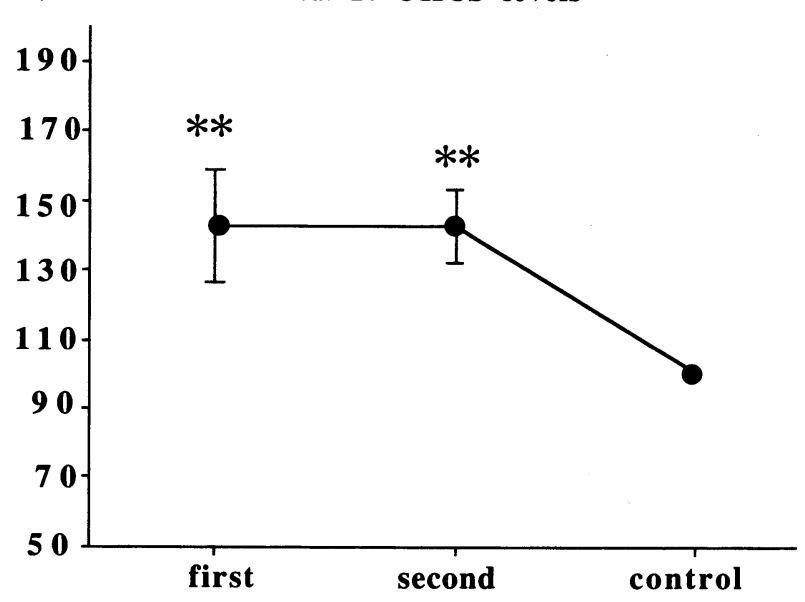

(\%)

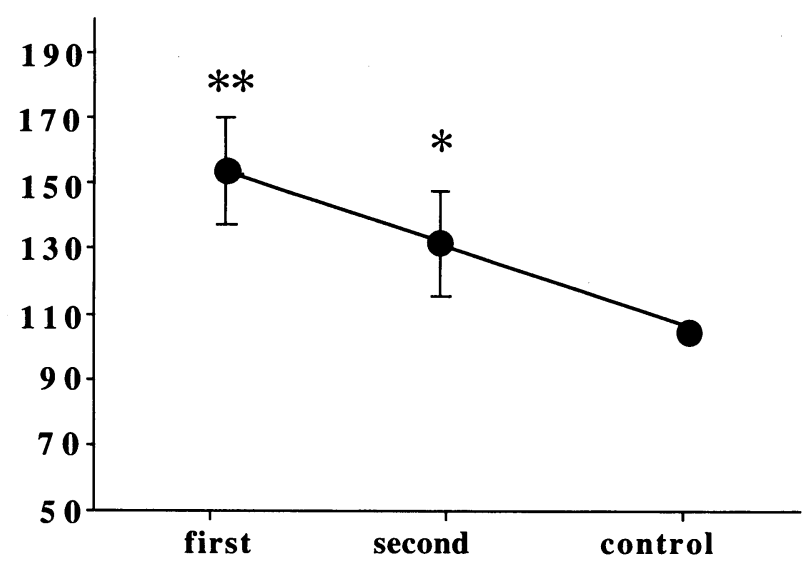

Fig. 4. Time course of 17-OHCS and 17-KS levels. $*$ : $\mathrm{p}<0.05^{* *}$ : $\mathrm{p}<0.01$ vs control $(\mathrm{n}=47)$

Each value is $\% /$ control. Error bar is Mean \pm S.E.M.

(\%)

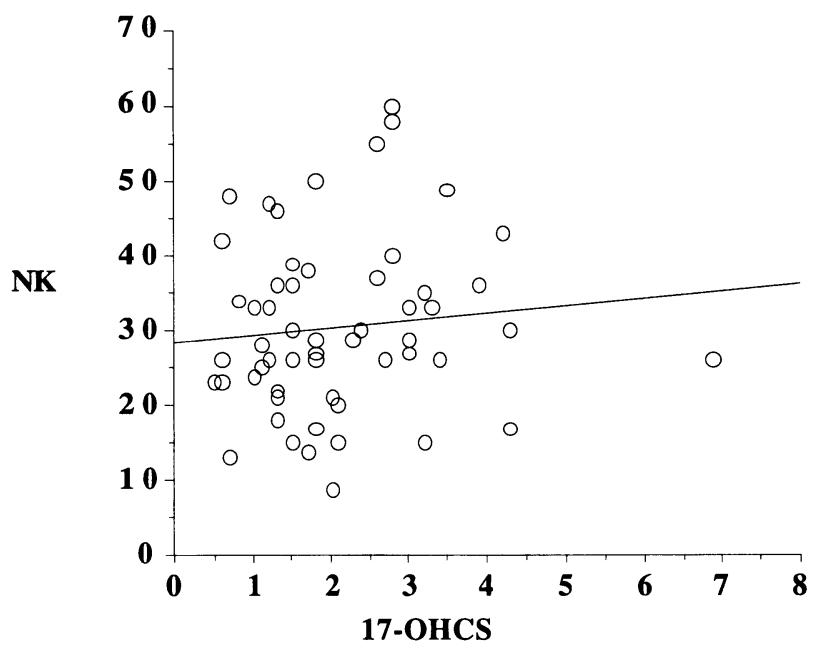

Fig. 5. Correlation between 17-OHCS and NK activity. $\mathrm{r}=0.1 \mathrm{p}=0.4601$

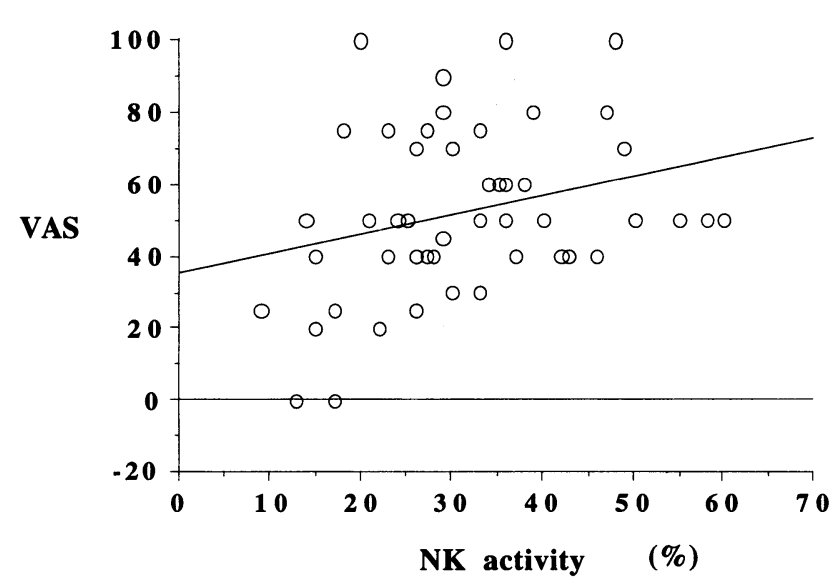

Fig. 6. Correlation between VAS values and NK activity. $r=0.269 \mathrm{p}=0.042$

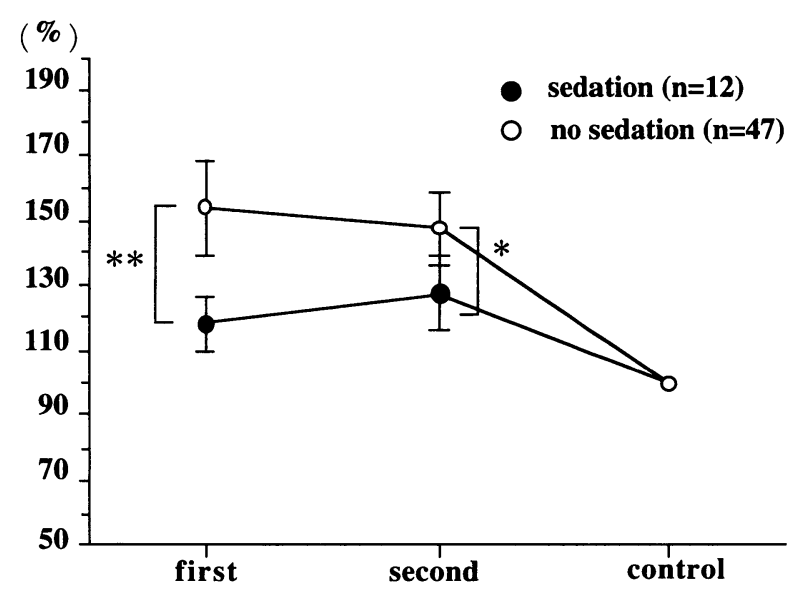

Fig. 7. Effect of ethyl loflazepate on 17-OHCS. ${ }^{*}: \mathrm{p}<0.05^{* *}: \mathrm{p}<0.01$

Each value is \%/control. Error bar is Mean \pm S.E.M.

\section{Results of a questionnaire}

The results of a questionnaire revealed that most patients noted an irritated feeling during the course of IMF. In addition, most patients replied that the inconvenience in eating was the most stressful issue during the course of IMF. Subsequently, when answers to the questions regarding an irritated feeling during the course of IMF were compared between ethyl loflazepate-treated and untreated groups, only 9 of 47 patients in the untreated group replied that they did not have an irritated feeling, whereas 13 of 47 patients replied that they had a potently or markedly irritated feeling. In contrast, 8 of 12 patients in the ethyl loflazepate-treated group replied that they did not have an irritated feeling, and 4 of 12 patients replied that they had a slightly irritated feeling. 


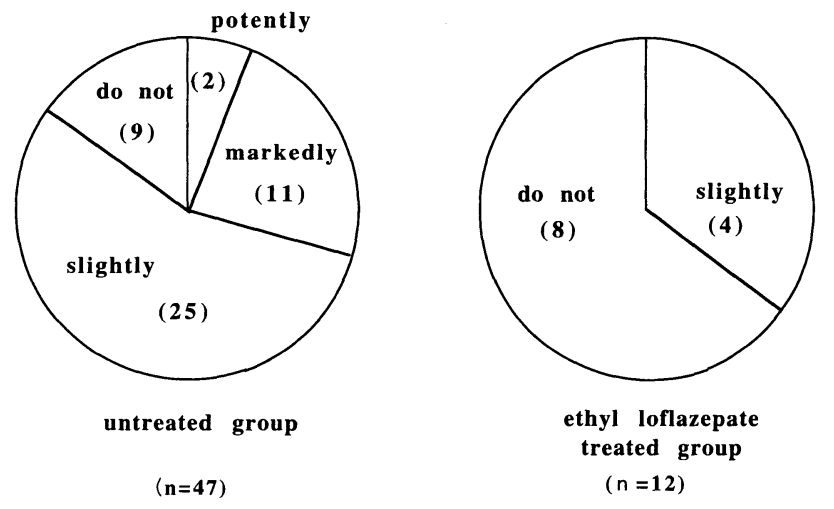

Fig. 8. Effect of ethyl loflazepate on irritated feeling.

However, no patients in the ethyl loflazepate-treated group reported potently or markedly irritated feelings (Fig. 8).

\section{DISCUSSION}

Currently, IMF is widely used to treat maxillofacial fracture, bone defects after the removal of cysts or tumors in the oral cavity, bone defects due to mutilation or segmental resection of the maxillary bone, or for gnathoplasty [19]. Physiologic mandibular movement is naturally lost in patients undergoing IMF, making food intake by mastication impossible and ordinary conversation difficult. The duration of IMF is approximately 1 month, but it can be shortened or sometimes prolonged for more than 2 months according to the patient's condition. Therefore, IMF can be extremely painful and very stressful to patients. It should be noted that the term, "stress" was originally used in the field of engineering to mean mechanical stress. Selye [20] contributed to the widespread use of the term "stress" in the field of medicine. Currently, the word, "stress" is frequently used as a generic medical term that means factors involved in the etiology and pathologic conditions of mental and physical disorders. Such a widespread use of the term, "stress" even in a daily conversation reflects the stressful modern society where various types of stress exist. Under such circumstances, psychological care for relieving patients from various types of stress is important in addition to the medical treatment of diseases. Selye [20] first proposed the concept of "stress" in 1936, and various studies have been conducted since. The recent advancement of neurochemistry, neuropharmacology, and immunohistochemistry revealed the presence of various neurotransmitters and neurotransmission modifiers in the brain. In addition, the development of microanalyses for these substances and their metabolites provided many novel findings regarding the intracerebral neurotransmission mechanism. Thereafter, many studies have been conducted to clarify the stress-induced biochemical changes in the brain [21-27]. Based on the theory that stress causes mental disorders such as psychosomatic disease and schizophrenia, and that brain noradrenergic neurons are closely involved in the pathologic conditions of these mental disorders, we evaluated changes in brain noradrenergic neurons under various types of stress. As a result, it was found that NA is involved in the action of adreno-cortical hormones via CRH $[28,29]$. Both 17-OHCS and 17-KS measured in this study are metabolites of the adreno-cortical hormone cortisol which are excreted in the urine. Therefore, it was considered that changes in intracerebral NA levels, that is, the severity of stress, can be estimated by measuring urinary17-OHCS and 17-KS levels [12].

In this study, the levels of 17-OHCS and 17-KS were significantly higher than the control values in patients undergoing IMF. However, the percent increase in 17-KS was lower than that in 17-OHCS, and 17-OHCS was positively correlated with stress caused by IMF. Therefore, it was suggested that $17-$ OHCS would be a useful parameter for evaluating stress in IMF.

Because NK cells play the principal role in nonspecific human immunity, NK activity is naturally decreased by various types of stress [30,31]. It was also reported that NK activity is decreased in patients with depression or chronic fatigue syndrome [32-34]. When NK activity in outpatients was measured in our Department, it was decreased in those who consulted our Department because of anxiety about malignant tumors, suggesting the involvement of psychological stress, such as anxiety or fear of cancer, in NK activity [35-37]. To clarify whether there is any difference in the various types of stress that decrease NK activity, we measured the levels of 17-OHCS and NK activity in patients undergoing IMF, and compared the results. However, as reported in this study, 17-OHCS levels were increased in patients who underwent IMF to treat fracture or deformity of the jaw during the course of IMF, but they were decreased after the release from IMF. In contrast, NK activity did not tend to increase even after the release from the stress of IMF. Moreover, there were no correlation between levels of 17OHCS and 17-KS, and NK activity $(r=-0.235)$. 
Although the prolonged duration of IMF becomes a chronic stress to the patients, NK activity did not significantly change in this study during the course of IMF. This finding suggested that a potent physical stress such as IMF only slightly influences the immune system because such stress is not closely associated with NK activity, showing the presence of various types of stress that affect the patient differently.

To explore a procedure for managing stress caused by IMF, the influence of an anxiolytic agent on 17-OHCS levels and the results of a questionnaire survey were evaluated in this study. Benzodiazepine anxiolytic agents such as ethyl loflazepate exhibit their actions over a prolonged period of time after binding with benzodiazepine receptors [38]. However, benzodiazepine receptors couple to $\mathrm{GABA}_{\mathrm{A}}$ receptors, a subtype of $\gamma$-aminobutyric acid (GABA) receptors, and thus exhibit their actions via $\mathrm{GABA}_{\mathrm{A}}$ receptor/benzodiazepine receptor/chloride ion channel supramolecular complex [39]. That is, the function of $\mathrm{GABA}_{\mathrm{A}}$ receptors is enhanced by the binding of ethyl loflazepate with benzodiazepine receptors, which increases the opening frequency of the chloride ion channel, resulting in hyperpolarization of the cell membrane and potent suppression of the stress. Suppression of stress via GABA $\mathrm{A}_{\mathrm{A}}$ receptors may play a leading part in the biochemical mechanism of the expression of anxiolytic and anticonvulsive actions of benzodiazepine agents [11].

Levels of 17-OHCS were significantly lower in patients treated with ethyl loflazepate during the course of IMF. In addition, a questionnaire survey revealed that the proportion of patients who complained of insomnia or of an irritated feeling was lower in those treated with ethyl loflazepate during the course of IMF, demonstrating the subjective antistress action of ethyl loflazepate.

These findings suggested that the prolonged duration of IMF is a chronic stress to patients undergoing IMF, and 17-OHCS is a useful parameter for evaluating the severity of stress in IMF. Moreover, 17-OHCS levels were significantly lower in patients treated with ethyl loflazepate during the course of IMF. Therefore, it was suggested that ethyl loflazepate relieves the stress caused by IMF.

ACKNOWLEDGMENTS: The author would like to be very grateful to Prof. Tadamitsu Kameyama, Dr. Chihiro Koga, Department of Oral Surgery, and Prof. Masatoshi Tanaka, Department of Pharmacology in Kurume University School of Medicine for their kind suggestion and correction of the manuscript.

\section{REFERENCES}

1. Koga C, Emoto H, Tanaka M, and Kameyama T. Effect of intermaxillary fixation (IMF) on rat brain noradrenaline turnover. Jpn J Oral Maxillofac Surg 1992; 38:1059-1062. (in Japanese)

2. Koga C, Emoto H, Yokoo H, Ishii H, Yoshida M et al. Effect of irritable stress model (intermaxillary fixation) on the rat immunological system and brain noradrenaline turnover. J Brain Sci 1999; 25:1-7.

3. Koga $\mathrm{C}$. The changes in noradrenaline release in the rat brain region caused by intermaxillary fixation (IMF) and their modifications by diazepam. J Kurume Med Assoc 1993; 56:145-157. (in Japanese)

4. Levy SM, and Herberman RB. Persistently low natural killer cell activity in normal adults: immunological, hormonal and mood correlates. Nat Immun Cell Growth Regul 1988; 8:173-186.

5. Pross HF, and Baines MG. Studies of human natural killer cells. In vivo parameters affecting normal cytotoxic function. Int J Cancer 1982; 29:383-390.

6. Masuda A, Nozoe S, Naruo T, Soejima Y, Nagai N et al. Natural killer cell activity in relation to psychobehavioral responses, stress coping behavior, and fatigue in healthy adult men. Jpn J Psychosom Med 1995; 35:383-390. (in Japanese)

7. Kusaka Y, and Morimoto K. Does lifestyle modulate natural killer cell activities? Jpn J Hyg 1992; 46:10351041. (in Japanese)

8. Nagao F, and Okumura K. Relation between stress and NK activity. J Clin Anesth 1997; 21:571-579.

9. Ohtani Y. A clinical study on natural killer (NK) cell activity in patients with cancer. J Jpn Soc Clin Surg 1983; 5:569-583. (in Japanese)

10. Tanabe K, Kinoshita Y, Tokuyoshi K, Houri D, Asagi K et al. Central nervous actions of ethyl loflazepate, a new benzodiazepine derivative. Yonago Acta Med 1982; 33:189-200. (in Japanese)

11. Mori A, Hata $Y$, Hasegawa $K$, Iwai H, Noguchi $T$ et al. Clinical study on CM6912 (ethyl loflazepate) in treatment of neuroses. Jpn J Clin Psychiat 1986; 15:275285. (in Japanese)

12. Koga C, Sakaino H, Fukuda K, and Kameyama T. Questionnaire investigation and biochemical examination about stress in intermaxillary fixation (IMF) patients. Jpn J Psychosom Dent 1996; 11:16-21. (in Japanese)

13. Kato T. 17-hydroxycorticosteroid (17-OHCS) and these fraction. Jpn J Clin Med 1985; 43 (A special Autumn issue): 896-901. (in Japanese)

14. Yakata $M$, and Sakurai A. Urinary 17-ketosteroid (17KS) and these fraction. Jpn J Clin Med 1985; 43 (A special Autumn issue): 914-916. (in Japanese)

15. Nagel JE, Collins GD, and Adler WH. Spontaneous or natural killer cytotoxicity of K 562 erythroleukemic cells in normal patients. Cancer Res 1981; 41:2284-2288.

16. Yasu K, and Sawada S. Target cell cytotoxic test. Medical Technol 1993; 21:574-580.

17. Ichihara K. Statistics for Bioscience. Nankodo Co., Ltd., Tokyo, 1990.

18. Ozaki M. Analysis of variance. In: Stat View 5.0, A 
Perfect Guide to Master, Nankodo Co., Ltd., Tokyo, pp158-184, 1999.

19. Assael LA, and Tucker MR. Management of facial fractures. In: Contemporary Oral and Maxillofacial Surgery, ed. Peterson LJ, Mosby Co., UAS, pp557-576, 1988.

20. Selye H. A syndrome by diverse nocuous agents. Nature 1936; 138:32.

21. Glavin GB. Stress and brain noradrenaline: a review. Neurosci Biobehav Rev 1985; 9:233-243.

22. Tanaka M, Kohno $Y$, Nakagawa R, Ida $Y$, Iimori $K$ et al. Time-related differences in noradrenaline turnover in rat brain regions by stress. Pharmacol Biochem Behav 1982; 16:315-319.

23. Glavin GB, Tanaka M, Tsuda A, Kohno Y, Hoaki Y et al. Regional rat brain noradrenaline turnover in response to restraint stress. Pharmacol Biochem Behav 1983; 19:287-290.

24. Iimori K, Tanaka M, Kohno Y, Ida Y, Nakagawa R et al. Psychological stress enhances noradrenaline turnover in specific brain regions in rats. Pharmacol Biochem Behav 1982; 16:637-640.

25. Nakagawa $R$, Tanaka $M$, Kohno $Y$, Noda $Y$, and Nagasaki N. Regional responses of rat brain noradrenergic neurones to acute intense stress. Pharmacol Biochem Behav 1981; 14:729-732.

26. Tanaka M, Kohno Y, Nakagawa R, Ida Y, Takada S et al. Regional characteristics of stress-induced increases in brain noradrenaline release in rats. Pharmacol Biochem Behav 1983; 19:543-547.

27. Tsuda A, Tanaka M, Ida Y, Shirao I, Gondoh Y et al. Expression of aggression attenuates stress-induced increases in rat brain noradrenaline turnover. Brain Res 1988; 474:174-180.

28. Emoto H, Koga C, Ishii H, Yokoo H, Yoshida M et al. A CRF antagonist attenuates stress-induced increases in NA turnover in extended brain regions in rats. Brain Res
$1993 ; 627: 171-176$.

29. Ganong WF. Neurotransmitter and pituitary function: regulation of ACTH secretion. Fed Proc 1980; 39:29232930.

30. Schedlowski M, and Schmidt RE. Stress and the immune system. Naturwissenschaften 1996; 83:214-220.

31. Abo T, Cooper MD, and Balch CM. Postnatal expansion of the natural killer and killer cell population in humans identified by the monoclonal HNK-1 antibody. J Exp Med 1982; 155:321-326.

32. Irwin M, Patterson T, Smith TL, Caldwell C, Brown SA et al. Reduction of immune function in life stress and depression. Biol Psychiatry 1990; 27:22-30.

33. Masuda A, Nozoe S, and Tanaka H. Psycho-behavioral responses and cellular immune function of adults, without any organic disorders, with chronic fatigue. Jpn J Psychosom Med 1993; 33:302-308. (in Japanese)

34. Mawle AC. Chronic fatigue syndrome. Immunol Invest 1997; 26:269-273.

35. Spiegel D. Cancer and depression. Br J Psychiatry (Suppl) 1996; 30:109-116.

36. Lesko LM. Supportive care and quality of life, psychological issues. Cancer: Principles \& Practice of Oncology 1997; 5:2879-2891.

37. Sato K. Immunological study on reactivation of latency infected herpes simplex virus type-1 in relation to natural killer-cell activity. J Kurume Med Assoc 1997; 60:19-27. (in Japanese)

38. Nakagawa T, Noda T, Kimura M, Fukamachi K, Sakata $\mathrm{T}$ et al. Clinical evaluation of ethyl Loflazepate (CM6912), a new antianxiety drug, on psychosomatic disorders and neuroses in the fields of internal and psychosomatic medicine -with special regard to the utility of administering CM6912 once daily-. Jpn Pharmacol Ther 1986; 14:281-298. (in Japanese)

39. Olsen RW. GABA-benzodiazepine-barbiturate receptor interactions. J Neurochem 1981; 37:1-13. 